\title{
Toward Autonomous Driving in Arctic Areas
}

\section{Ruotsalainen, Laura}

2020

Ruotsalainen , L, Renaudin , V , Pei , L , Piras , M , Marais , J , Cavalheri , E \& Kaasalainen , S 2020 , ' Toward Autonomous Driving in Arctic Areas ', IEEE Intelligent Transportation Systems Magazine , vol. 12 , no. 3 , pp. 10-24 . https://doi.org/10.1109/MITS.2020.2994014

http://hdl.handle.net/10138/326363

https://doi.org/10.1109/MITS.2020.2994014

unspecified

acceptedVersion

Downloaded from Helda, University of Helsinki institutional repository.

This is an electronic reprint of the original article.

This reprint may differ from the original in pagination and typographic detail.

Please cite the original version. 


\title{
Towards Autonomous Driving in Arctic Areas
}

\author{
Laura Ruotsalainen, Valerie Renaudin, Ling Pei, Marco Piras, Juliette Marais, Emerson Cavalheri \\ and Sanna Kaasalainen
}

\begin{abstract}
This review article provides an overview of the use of inertial and visual sensors and discusses their prospects in arctic navigation of autonomous vehicles. We also review the fusion algorithms used so far for integrating vehicle localization measurements as well as the map matching algorithms relating position coordinates with the road infrastructure. The review reveals that the conventional fusion and map matching methods are not enough for navigation in challenging environments, like urban areas and Arctic environment. We also provide new results from testing inertial and optical sensors in vehicle positioning in snowy conditions. We find that the fusion of Global Navigation Satellite Systems (GNSS) and Inertial Navigation System (INS) does not provide the accuracy required for automated driving, and the use of optical sensors is challenged by snow covering the road markings. While extensive further research is needed to solve these problems, fusion of GNSS, INS and optical sensors seems to be the best option due to their complementary nature.
\end{abstract}

Index Terms-Arctic navigation, autonomous driving, fusion algorithms, optical positioning

\section{INTRODUCTION}

A UTONOMOUS driving is already here, and replacing human drivers by self-driving vehicles is expected to be reality in the next 10 years [35]. The evolution of autonomous driving can be seen as a continuation of technology development started out from driver assistance systems [6]. Extensive research carried out for intelligent transport and self-driving vehicles has been carried out from different viewpoints over the years, such as sensor systems, robotics, artificial intelligence, and socioeconomic modeling [7], [9], [83]. Numerous research organizations and car manufacturers have developed prototypes, and an increasing number of tests are already taking place in public roads (e.g., [1], and refs. therein). Automated vehicles set much higher demands for positioning accuracy and especially integrity. While meter level accuray is enough for personal vehicle navigation, automated vehicle require centimetre accuracy and very high integrity [61].

Global Navigation Satellite Systems (GNSS), provide good performance in open outdoor environments. However, various error sources affect the accuracy and reliability of the position solutions computed using the GNSS signals and especially in urban canyons GNSS is significantly degraded or unavailable. For overcoming the aforementioned navigation challenges, research has been very active for decades for developing novel improved signal processing techniques [75], [27] and finding a suitable set of other methods for augmenting or replacing the use of GNSS. The size, price and power consumption requirements set for the system complicate the method development further, and therefore the set of equipment, error modelling and

Submitted to review on $9 / 2018$

L. Ruotsalainen is with Department of Computer Science, University of Helsinki, and Finnish Geospatial Research Institute, Finland. V. Renaudin and Juliette Marais are with IFSTTAR, France, Ling Pei is with Shanghai Key Laboratory of Navigation and Location-based Services, School of Electronic Information and Electrical Engineering, Shanghai Jiao Tong University, Shanghai, China. M. Piras is with Politecnico di Torino, DIATI - Department of Environment, Land and Infrastructure Engineering. M. Cavalheri is with Department of Geodesy and Geomatics Engineering, University of New Brunswick, Canada.

Corresponding authors: Ruotsalainen Laura (e-mail: author@ helsinki.fi), Ling Pei (email: ling.pei@sjtu.edu.cn) algorithms used for integrating measurements from different sources, has to be considered carefully.

Due to the GNSS limitations in harsh environments hybridization with other sensors, in particular with Inertial Navigation Systems (INS) has been used in positioning for the road environment since decades. However, competitive and complementary positioning technologies are fast developing and opening new frontiers for hybridized and cooperative positioning. The scope of such integration is not only to improve accuracy and precision of the solution, but also to provide means to increase robustness and reliability of the procedure to threats typical of the urban environment, such as signal obscuring, multipath and radio-frequency interference. Positioning based on utilization of wireless networks has become popular in recent years especially in dense urban and indoor environments. These terrestrial positioning technologies are being consolidated and several works for the hybridization with GNSS core positioning is being studied, following to a certain extent the integration process of GNSS and INS. However, research is still needed to define proper error models, tailored integration algorithms and other topics to fully exploit the diverse source of information.

Cameras are increasingly used for positioning for a wide field of applications at all levels of accuracy. The success of optical methods originates from miniaturization and advancement in the technology of detectors (e.g. charge-coupled device CCD sensors). In parallel there has been an increase in data transmission rates and computational capabilities of processing equipment as well as profound development of algorithms in image processing. On intelligent vehicles, cameras are of high interest to be used independently or, preferably, hybridized with GNSS, since they are equipping more and more frequently the new vehicles and therefore can provide valuable information at a very low additional cost. Last but not least light detection and ranging (LiDAR) can be an attractive technology for positioning due to its high accuracy in ranging, wide area view and low data processing requirements [60].

An important aspect to autonomous driving is the technology to be used in sensing the environment. Use of laser scanners and radars have been studied for years as means 
of object detection, even for harsh weather conditions [37] [38]. However, laser scanners are still expensive and therefore more cost effective means should be considered. Sensors are not only important for the perception of the environment, i.e., detecting obstacles, traffic and lane markers, road conditions, and other road users, but also for mapping and localization, since the limitations of GNSS for this purpose are well understood, especially in dense urban areas [9].

Most of the testing of sensor and localization systems so far has been carried out in summer conditions: either on public highways [1] [78] or test platforms, such as the Mcity by the University of Michigan [48] [69]. Testing self-driving cars in arctic conditions has started only recently, with a few projects ongoing. One of these has been started by the Google's selfdriving car, Waymo [78]. VTT Technical Research Centre of Finland made the first self-driving tests in Finland on snowy conditions [79]. Their vehicle is relying on forward sensing laser scanners and radar, as well as cameras. The winter tests were carried out in the E8 Aurora intelligent road, which is a public test ecosystem in the Finnish Lapland for testing autonomous vehicles in all conditions, especially in winter with snow and ice present [4]. As yet, there is not enough information on the performance of the localization and navigation systems in snow conditions for autonomous vehicles. Little or no test results have been published so far. These conditions are challenging, since snow often covers distinctive features, such as lane markings, and weather conditions restrict or even obstruct the visibility. More research and testing is necessary to find the optimum combination and settings of sensors, and to make autonomous cars reliable in all conditions.

In this paper, we review the methods related to autonomous vehicle navigation and discuss their challenges at the arctic environments concentrating especially on optical sensors. We have tested the use of inertial sensors and a camera in arctic winter to emphasize challenges related to their use in snowy conditions. To our knowledge, this is one of the first research efforts to summarize and assess the feasibility of inertial and camera sensors for this purpose.

This paper is structured as follows. In Section III we discuss the state-of-the-art technologies for autonomous vehicles and their challenges in arctic environment. In Section III we first review the different optical positioning methods and their challenges in snowy conditions, and provide new results from testing the feature detection capabilities of two widely used detectors. Section IV discusses the different estimation algorithms feasible for fusing the different measurements into an accurate and reliable position solution. Finally, conclusions and discussion of areas of future work are in Section VI]

\section{TEChNOLOgIES ENABLING AUTONOMOUS DRIVING}

Arctic areas set special challenges for GNSS positioning. The nominal accuracy of GNSS positioning, 5-10 meters in open areas, is not sufficient for autonomous vehicles. Therefore, augmentation systems both ground and satellite based are used for improving the accuracy down to sub-meter level. Arctic areas are often sparsely populated and therefore the infrastructure enabling ground based augmentation does not exist. Also, due to the constellation characteristics of for example the European Geostationary Overlay Service (EGNOS) Space Based Augmentation Systems (SBAS) do not provide sufficient augmentation performance [8]. Therefore, fusion of GNSS and other positioning means carried by the vehicle by itself is the only feasible solution for Arctic autonomous driving.

Fusion of GNSS and inertial sensors is an attractive setup for positioning due to their complementary characteristics and prevalence in automated vehicles. Inertial sensors are used to provide or improve navigation solution when GNSS is not available or degraded. Inertial sensors do not provide absolute position, but speed and direction measurements, which may then be used for extrapolating the last known absolute position. This process is called Dead Reckoning (DR) [31]. However, snow conditions cause slipping of the vehicle that is difficult to estimate using low-cost inertial sensors. The bias in position estimate caused by slipping is smaller with inertial sensors than with the wheel speedometers usually used for estimating the speed of the vehicle. In addition to sliding effects, multipath and fading effects deteriorate the satellite based positioning solution in challenging environments, even in forests covering large parts of the Arctic region.

Initial testing of low-cost commercial navigation system fusing GNSS and inertial sensors was conducted in Finland in snowy arctic conditions in March. The data collection lasted for 25 minutes and the route consisted of large highways (Fig 1) as well as smaller forest roads (Fig 2). A MTi-G-700 GNSS/INS hybrid receiver from XSens provided the fused position solution. Novatel SPAN system, providing centimetre level accuracy, was used as a reference. The hybrid receiver and the span reference system were rigidly attached together located behind the passenger seat. The same GNSS antenna, located on the top of the car roof, was used by both systems. Fig 3 shows the system configuration inside the vehicle. The setup also included a GoPro camera installed behind the wind shield. Analysis of the effect of snow into optical positioning will be discussed in Section

The GNSS and INS measurements were fused using a tightly-coupled Extended Kalman filter. The obtained navigation solution is degraded in snowy conditions. The 50\% cumulative horizontal position error in our test was $5.14 \mathrm{~m}$ and $95 \% 14.96 \mathrm{~m}$. With the high accuracy demands set by autonomous driving, it is evident that the fused GNSS/INS solutions is not accurate enough. However, navigation using a fused GNSS and INS solution alone does not provide enough accuracy for autonomous driving with centimetre level accuracy requirements [14] even in non-arctic conditions. More sensors are needed for providing all information required to provide sufficiently accurate and reliable solution.

Technologies enabling autonomous driving may be roughly divided into hardware (different sensors) and algorithms (for fusing sensor measurements and for maturing position information into navigation, routing and decision information). McGehee et al. [49] have defined the state-of-the-art autonomous vehicle technologies to be radar, ultrasonic sensor, GNSS, dedicated short range communication, cameras, 


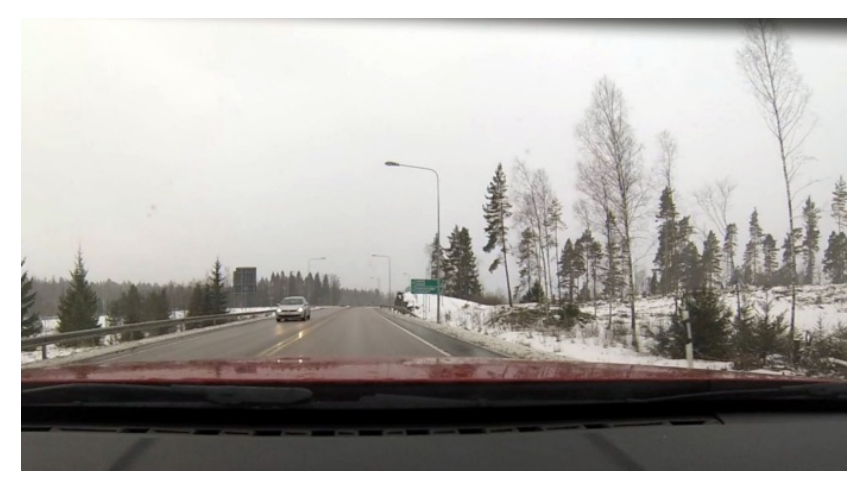

Fig. 1: Test environment 1, highway

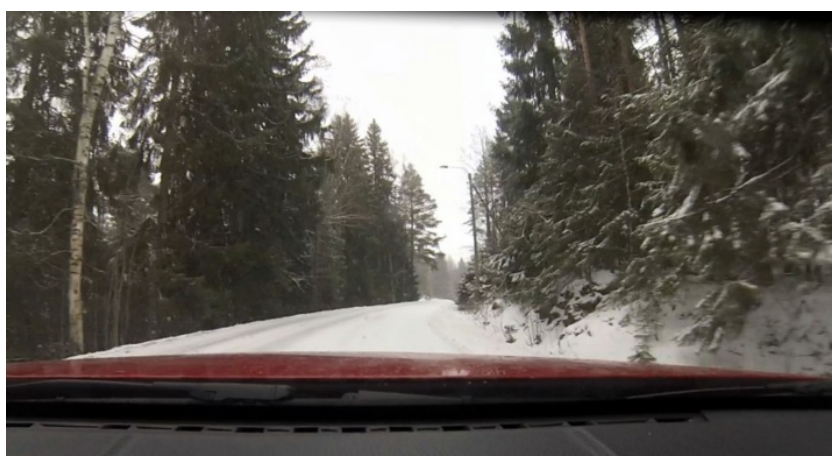

Fig. 2: Test environment 2, forest

LiDAR, sensor fusion, mapping technologies, route planning and navigation algorithms and localization, object detection and mapping. Table $\mathbb{I}$ gives a short review of the functionalities the hardware technologies provide and what kind of challenges they experience at the Arctic environment.

The autonomous vehicle technologies listed contributing to the position computation are LiDAR, GNSS and cameras. At arctic areas especially ice sets challenges for LiDAR, because the shiny ice causes the laser pulses to careen around and therefore does not provide an accurate solution. Later sections provide more detailed evaluation of the use of cameras in the arctic environment and discusses how sensor fusion and map matching should be implemented to provide a viable end solution.

\section{OPTICAL POSITIONING}

The use of cameras in positioning is facilitated by the decrease of their size and costs and their advantage is that they provide accurate motion measurements and relative positioning means. There are two different approaches to camera-based positioning. The first one uses a camera as a means to detect visual features in a close surrounding environment and obtains relative positioning information by detecting the motion of the features in images [24]. This approach is also called visionaiding. The motion of the features enables computation of attitude and translation of the camera between consecutive images. This motion information may be further integrated with e.g. GNSS or other sensor measurements to obtain complementary information for positioning. The information
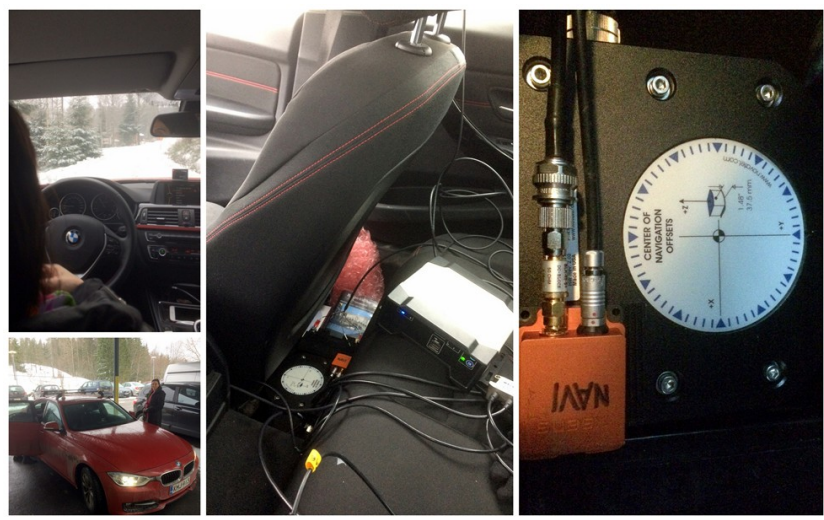

Fig. 3: Test equipment. Left up: GoPro camera on the wind shield, left down: antennas on the vehicle's roof. The hybrid receiver and the SPAN reference system were rigidly attached together (right) and located behind the passenger seat (middle)

TABLE I: Autonomous Vehicle Technologies

\begin{tabular}{|l|l|l|}
\hline Technology & Used for & Arctic-specific challenges \\
\hline Radar & $\begin{array}{l}\text { Ranging for park assis- } \\
\text { tance, collision warning }\end{array}$ & $\begin{array}{l}\text { Does not work if covered } \\
\text { with snow or ice }\end{array}$ \\
\hline $\begin{array}{l}\text { Ultrasonic sen- } \\
\text { sors }\end{array}$ & $\begin{array}{l}\text { Distance to objects for } \\
\text { backing, parking and } \\
\text { lane keeping }\end{array}$ & $\begin{array}{l}\text { Does not work if covered } \\
\text { with snow or ice }\end{array}$ \\
\hline GNSS & $\begin{array}{l}\text { tion and velocity solu- } \\
\text { tions position sol coverage of augmen- }\end{array}$ & $\begin{array}{l}\text { Poor } \\
\text { tation systems, both ground } \\
\text { based and satellite based }\end{array}$ \\
\hline $\begin{array}{l}\text { Dedicated } \\
\text { Short Range } \\
\text { Communica- } \\
\text { tions (DSRC) }\end{array}$ & $\begin{array}{l}\text { For communication be- } \\
\text { tween vehicles (V2V), } \\
\text { and vehicle to infrastruc- } \\
\text { ture (V2I) }\end{array}$ & $\begin{array}{l}\text { No arctic specific challenges. } \\
\text { Temperature, humidity, and } \\
\text { wind are not correlated to } \\
\text { radio frequency (RF) propa- } \\
\text { gation loss at a statistically } \\
\text { valid level of significance } \\
\text { [46] }\end{array}$ \\
\hline Cameras & $\begin{array}{l}\text { Optical positioning, ob- } \\
\text { ject recognition }\end{array}$ & $\begin{array}{l}\text { Snow and ice distort or pre- } \\
\text { vent feature and object detec- } \\
\text { tion }\end{array}$ \\
\hline LiDAR & $\begin{array}{l}\text { Distance and angle to } \\
\text { object surrounding the } \\
\text { vehicle, used for posi- } \\
\text { tioning and mapping }\end{array}$ & Reflections caused by ice \\
\hline
\end{tabular}

provided by the images does not suffer from accumulating measurement errors typical for inertial sensors, or signal obstruction disturbing GNSS processing. Related theory and relevant applications will be discussed in Section III-B

The second approach uses image processing in order to help the GNSS receiver to mitigate the degradation of the position solution due to inclusion of Non-line-of-Sight (NLOS) signals. Exclusion of NLOS signals improves GNSS performance in environments where surrounding objects obstruct the signals, for example buildings in urban areas. Some of such methods compare 3D databases to the recorded image [12] or inputs extracted from images such as skyline [59]. The first NLOS mitigation methods relied on classical linear cameras [45], while the more recent ones rely on a fish-eye lens pointed up over the roof of the vehicle [44], [20]. NLOS mitigation using optics will be discussed in Section III-C

The drawbacks of cameras is that, a calibration process is required for applications that need very accurate mea- 
surements. There is also a need for the lenses to be clean and sensitive to light variations that make generic processing algorithm difficult especially during the night and in rain or snowfall. The use of cameras will benefit in the near future from both technological improvements in hardware as well as the development of more robust, fast and efficient processing solutions. However, the mitigation methods for the effects of snow on road and snowfall are to be assessed.

\section{A. BASICS OF OPTICAL POSITIONING}

Photogrammetry allows to generate $2 \mathrm{D}$ or $3 \mathrm{D}$ model of a scene using images or frames, to recover the exact positions of surface points. Photogrammetry is based on collinearity equations [55], where the distance between two points that lie on a plane parallel to the photographic image plane, can be determined by measuring their distance on the image, if the scale (s) of the image is known. Traditionally, photogrammetry is based on a pair of images, which could be collected in two different ways: 1) using two or more simultaneous cameras or 2) using only one camera but collecting images from different points, with a specific relative distances, in order to respect the scale factor and the overlapping (at least $60 \%$ on longitudinal direction and $40 \%$ on transversal one). These approaches are adopted both in aerial and terrestrial applications, the selection is made considering the object, the additional tools (e.g. ground control points) and instruments used for navigating.

In Visual Based Navigation (VBN), the boundary conditions are completely different because ground control points are not available in an unknown environment or VBN is used for augmenting or replacing other navigation systems. Also, low quality of cost-effective cameras used on vehicles and lack of distinctive features in images representing a large portion of the road and sky complicate the photogrammetric observations. In fact, all digital cameras introduce several deformations into images due to the lens and the sensor. Considering the optical lens, there are two main types of distortion: radial and tangential.

Traditionally, the device used for $\mathrm{VBN}$ in a vehicle is an integrated non-metric camera. Calibration is required through analytical procedures to study the characteristics of the mechanical-digital system, to evaluate the distortion parameters and other errors. In particular, the calibration aims to evaluate the effects of radial, tangential and affine distortion of the sensor [25]. Most mass market cameras use the selfcalibration procedure, which is based on the determination of the calibration parameters carried out independently by photogrammetric survey. This is usually performed by preparing a calibration grid, specifically made, in which the coordinates of the target are known with high precision.

The vocabulary related to optical perception in navigation is manifold. Computer vision deals with the algorithms used also for close range photogrammetry, whereas the term machine vision is concerned with the use of computer vision in industrial or practical processes [21]. Structure From Motion (SFM) recovers relative camera poses and three-dimensional (3-D) structure from a set of camera images (calibrated or noncalibrated) [41], [24]. Visual odometry is a particular case of
SFM concentrating on resolving the camera 3D ego-motion sequentially in real-time, when SFM additionally reconstructs the structure of the scene [72]. Bundle adjustment [19] can be used to refine the local estimate of the trajectory. Here we use the term optical positioning for the process of obtaining relevant information from images and visual based navigation to compute the navigation solution while using the optical positioning information alone or fused with other measurements.

\section{B. solutions for OPTICAL POSITIONING challenges}

[70] gives an extensive review of the history and relevant technologies for estimating vehicle's motion from visual input alone. This research started already in the early 1980s [53]. The first methods used stereo cameras, where the relative 3-D position of the features was directly measured by triangulation to derive the relative motion. However, camera's size sets constraints for the use of stereo perception. When the baseline, namely the distance between two cameras in the stereo setup, is much smaller than the distance between the camera pair and the object in the scene, the resulting triangulation does not provide correct measurements. Therefore, it is attractive to use monocular cameras in VBN. The disadvantage is that they can provide the motion only up to a scale factor. Different approaches have been developed for determining the absolute scale, for example using constrained configuration of the camera, fusing inertial or range sensors in VBN system or using known characteristics of the navigation environment, namely sizes of elements in the scene.

The early stereo camera solutions did not provide the required accuracy. The first real-time method with a robust outlier rejection scheme RANdom SAmple Consensus (RANSAC) was presented 2004 [55]. Ultra-tightly coupled GNSS /INS and optical positioning providing good accuracy and reliability for vehicle positioning also in urban areas was presented in [3]. In monocular VBN the absolute scale is unknown and traditionally the distance between the first two camera poses has been initialized to one. As a new image arrives, the relative scale and camera pose with respect to the first two frames are determined using either the knowledge of scene dimensions or the trifocal tensor. Trifocal tensor resolves the scale using the three images by comparing all projective geometric relationships among three views depending only on the relative motion of the camera among the views [24].

Optical methods used for positioning can be divided into three categories: feature-based methods, appearance-based methods, and hybrid methods. Feature-based methods are based on salient and repeatable features that are tracked over the frames. Appearance-based methods [22], [52] use the intensity information of all the pixels in the image or its subregions, and hybrid methods use a combination of the previous two [71]. However, appearance-based approaches are not robust to occlusion. This is a major disadvantage especially for vehicle applications, where other vehicles occlude the scene frequently. A sophisticated hybrid solution was presented in [72], where appearance was used to estimate the rotation of the car and features from the ground plane to estimate the translation and the absolute scale. Recently, [77] has used the 
camera for lane detection intended especially for autonomous vehicles.

Motion of the vehicle is constrained and the knowledge of the motion constrains enables methods with decreased computation time and improved accuracy. The most used motion constrain is the knowledge of the static camera height and attitude. This allows to use a camera facing the road surface. Then, a method called homography provides motion estimates with an absolute scale [39], [32], [84]. A method for mitigating the motion constraint requirements was developed in [66]. It was aimed mainly for pedestrian navigation, but it was also applicable for vehicles in urban areas.

\section{NON-LINE-OF-SIGHT MITIGATION USING OPTICS}

When included into GNSS position computations, NLOS signals degrade the obtained accuracy. [10] classifies the assessment of NLOS problem into four different levels; NLOS can be ignored, mitigated, identified or avoided. In land transportation, ignoring or avoiding the NLOS will not provide required performance. Optical methods can help identify the presence of NLOS by detecting environmental obstacles around the receiver antenna but also to identify the satellites concerned thanks to the comparison of their position and of the obstacle positions.

A direct signal is received when the path between the satellite and the antenna is above a visibility line, also called optical horizon line. Optics was used first in telecommunication studies to detect satellite visibility [64]. In the first implementations of NLOS mitigation methods two video cameras were looking left and right of a vehicle path [45]. State-ofthe-art methods use fish-eye lens cameras capable of showing the complete horizon above the receiver antenna [44] [20] as illustrated in Fig 4. A similar approach was proposed by [50] with an infrared (IR) camera.

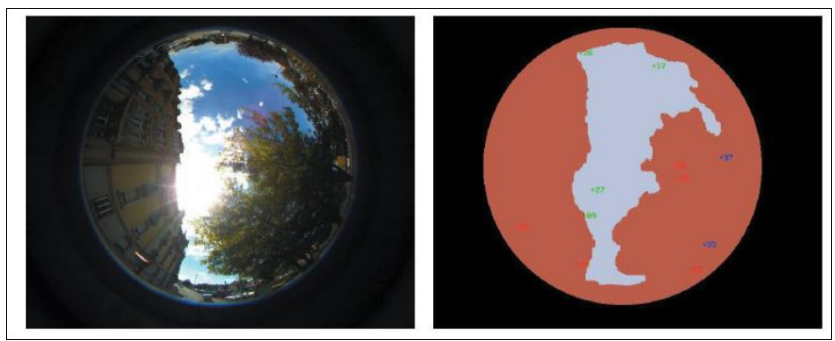

Fig. 4: Fish-eye image acquired on the top of a road vehicle and processed in order to detect masking and sky areas [43]

Once identified, NLOS mitigation can rely on different strategies : the first one relies on excluding the reflected signal from the position estimator as tested by [73]. However, in some conditions, the image-based exclusion can been too severe and degrade accuracy because of a poor resulting geometry of the satellites [44]. [68] proposed the use of a camera to detect and exclude NLOS in a GNSS/INS/Fish-eye camera integrated system.

The second strategy aims to adapt the solution computation in order to benefit from the measurement but minimizing the error which is in this case inclusion of NLOS signals. In [76] and [43], NLOS optical results were inputs for a weighting schemes. The main idea is that detected NLOS signals will be represented with lower weights than LOS signals in the least square estimator and therefore the measurements obtained using LOS signals contribute more into the position computation resulting in better position accuracy. With the dataset used in [43], the mean error was $3.28 \mathrm{~m}$ with the NLOS detection based on images, but $10.58 \mathrm{~m}$ with a classical least square or $6.5 \mathrm{~m}$ with an extended Kalman Filter. Most of the papers addressing the NLOS mitigation have been based on optics used in dense urban environments where the GNSS signal is most degraded. However, snowfall will cause major challenges for obtaining relevant information with upward facing cameras.

\section{STUDYING THE EFFECT OF SNOW ON OPTICAL POSITIONING}

Feature based optical positioning is based on tracking features of static objects and thereby computing vehicle's motion. In order to find reliably static objects they should be extracted close to the road surface. Many of the car manufacturers pioneering on automated vehicle development compute the motion using lane marks as features [77]. However, snow conceals most of the features on the road surface. As stated above, there are no studies published about the challenge snow causes for optical positioning.

To underline the importance of considering the effect of snow on optical positioning we collected images during the data collection campaign described in section $\Pi$. We studied the feature detection capabilities of two widely used feature detectors; Hough Transformation [26] for detecting lines from images and Features from accelerated segment test (FAST) algortihm [15] for detecting point features. All images were processed using Matlab. Fig. 5 shows the situation for detecting FAST features in a good road condition, namely there were hardly any snow on the road surface. The figure overlays two consecutive images (thereby the color of the image is distorted at some points and positions of objects in the first image are shown with turqoise) and shows the detected and matched features using a red cross (first image) and yellow circle (second image). Outlier were discarded using the RANSAC algorithm [16]. The figure shows an adequate number of reliable matched features, which allows for accurate optical vehicle positioning. Fig. 6 shows the result for line detection at the same location. Lane markings are well found from the road surface. However, when the road surface is even slightly covered by snow and ice, the situation is completely different, as can be seen from Fig. 7 There are no matching features found from consecutive images close to the road surface. Also, lane markings are not detected by Hough Transformation. Hough Transformation is a feasible line detector in most environments, but based on our tests it is clear that more sophisticated means for feature detection, for example based on Convolutional Neural Networks (CNN) [82] in arctic conditions is required.

Snowfall and rain set additional challenge for optical positioning. In the case of light snowfall or rain the challenge 
is to avoid tracking dynamic flakes or drops. Light snowfall was experienced during our data collection campaign. Fig 8 shows the result of detecting and matching FAST features from the image when the vehicle was static. All crosses, i.e. features detected from the second, are in the middle of the red circle, i.e. features detected from the first image. This means that only static features are tracked between the two images. Therefore, it is shown that no flakes in the air or rain drops on the windshield are detected by the detector. Therefore, light snowfall or rain do not disturb the process. However, when a snowstorm or heavy rain is experienced the situation changes. A lot of research has been done to identify and remove the flakes and drops from the image keeping the background, e.g. [58] [57]. However, when the snow or rain cover most parts of the scene or gather to the camera lens, optical positioning becomed impossible. Similarly, LiDAR processing becomes impossible due to the reflections caused by snow and rain. The solution for assuring safe autonomous driving in most hars conditions, in snow storm or very heavy rain does not yet exist. When snowflakes or heavy rain dominate the view of cameras and LiDARs, their performance will be too degraded for providing positioning. Although novel sensor technologies are continuosly developed, like a radar able to scan below snow cover and road, it is forecasted that the emerge of technologies able to cope with these most demanding weather conditions will take at least until 2020 [11].

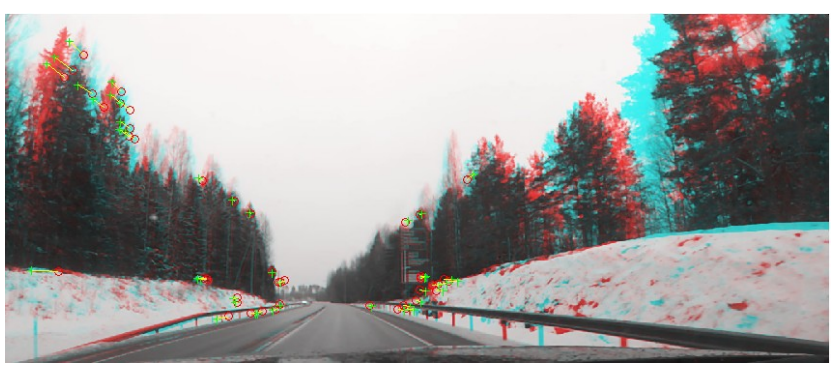

Fig. 5: Matching FAST features detected from consecutive images in good weather condition. Features shown using a red circle for the first image and yellow cross for the second.

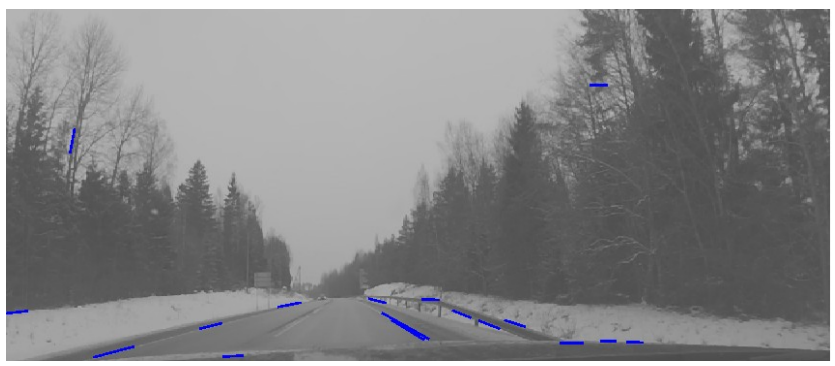

Fig. 6: Lines detected (blue) from the image using Hough Transform

\section{FUSION ALGORITHMS}

It is self-evident that due to the characteristic challenges faced by different positioning technologies, no individual

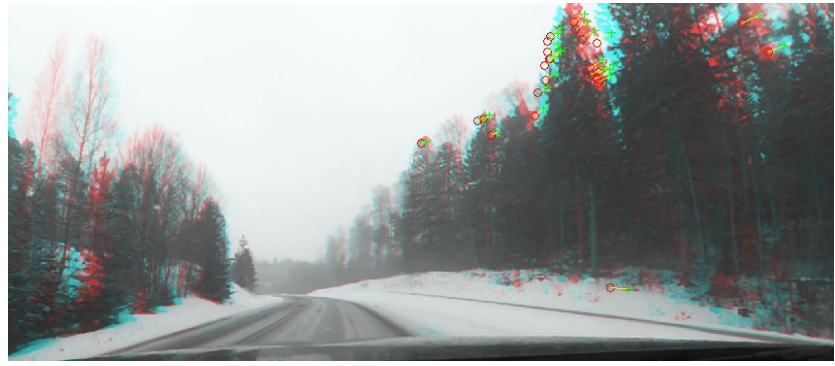

Fig. 7: Matching FAST features detected from consecutive images from snowy and icy road. Features shown using a red circle for the first image and yellow cross for the second.

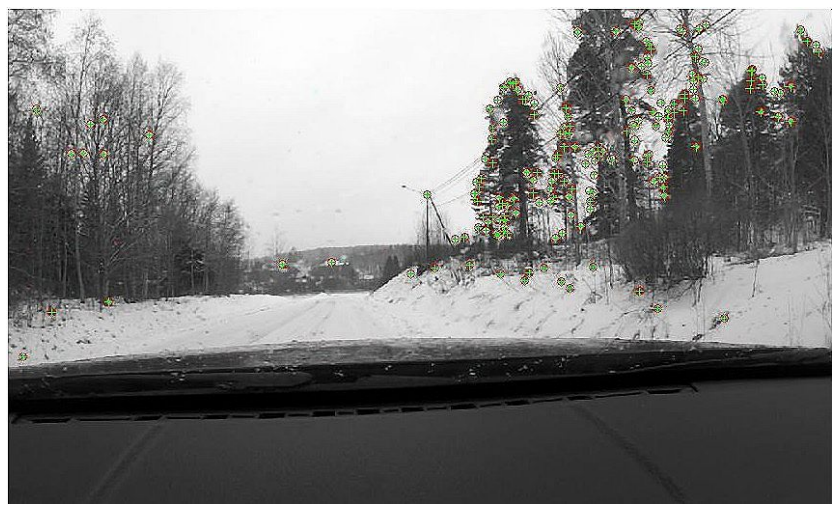

Fig. 8: FAST features detected and matched from two images taken in snowfall (the contrast has been increased to get the snowfall and water on the wind shield visible in the image)

technology can provide the accuracy and reliability required for positioning of autonomous vehicles. Therefore, the fusion of measurements with often very different nature and error characteristics is needed. This section provides the reader with a review on the fusion algorithms suitable for vehicle positioning.

\section{A. WEIGHTED FUSION ALGORITHM}

The weighted fusion algorithm is the simplest and most intuitive fusion algorithm. In the weighted fusion algorithm, position information provided by multiple fusion sources is given weights to obtain the final fusion results as shown in Fig $9 f_{1}(x), f_{2}(x), \ldots, f_{n}(x)$ are the position results computed by each sensor involved, $w_{1}, w_{2}, \ldots, w_{n}$ are the corresponding weights and $F(x)$ is the resulting fused position solution. Weights are tuned via experimental testing and result analysis for each sensor. Although implementation of the weighted fusion algorithm is simple, accuracy of the fusion result depends on the goodness of the selected weight factors. Therefore, the process of weight tuning based on large number of tests is the most important part of the algorithm implementation.

\section{B. KALMAN FILTERING}

Kalman Filter (KF) algorithm uses a state space equation and measurement model recursion to derive the optimal fusion data state estimate. Statistical estimation and the known 


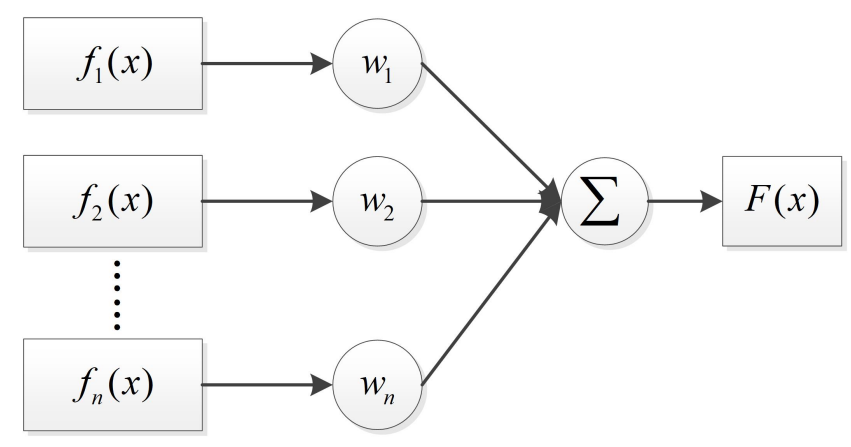

Fig. 9: Diagram of the weighted fusion algorithm

mathematical system model are used [30]. The main five key elements of KF are the state estimate, state covariance matrix, system model, measurement vector, and measurement model.

When fusing GNSS and INS, INS error model is used as the system model. In the easiest form of Kalman filtering (loosely-coupled), the data is fused in the position domain and the measurement vector is the position difference between observations of INS and GNSS.

The system model is dependent on the system's motion states and environment's characteristics. For example, autonomous vehicles operating at the polar region have poor GNSS satellite geometry, presented using a measure called Geometric Dilution of Precision (GDOP) [31], resulting in large measurement error. Kalman filtering is intended for estimating linear processes, however autonomous driving is a nonlinear problem. Typically, extensions of Kalman filtering, namely Extended Kalman Filter (EKF) and Unscented Kalman Filter (UKF) are applied to deal with problems of nonlinear systems [28]. Motion model of an autonomous vehicle has many constraints such as little changes along the vertical axis. Therefore, constrained Kalman Filter (CKF) [23] can be utilized for improved performance over the non-optimal linearization solutions obtained using EKF and UKF. In addition to data fusion, Kalman Filtering has been widely used for example for calibrating INS [34] and GNSS signal processing [5].

In a complex system, such as an autonomous vehicle processing heterogeneous and asynchronous data from multiple sensors, a centralized Kalman filter is not robust enough. As shown in Fig 10. Federal Kalman filter is a decentralized filter usually applied for complicated systems. It can solve the challenge of estimating the state with high dimensional computation, good fault tolerance and flexible design [81].

\section{PARTICLE FILTERING}

The idea of particle filtering is derived from the Monte Carlo method [2]. Particle filtering extracts random state particles (also called samples) from the posterior probability, using a method called Sequential Importance Sampling (SIS). The state-space model can be non-linear and the initial state and noise distributions can take any form required. The method can transform complicated integral operations into summation operations of finite sample points and provides improved

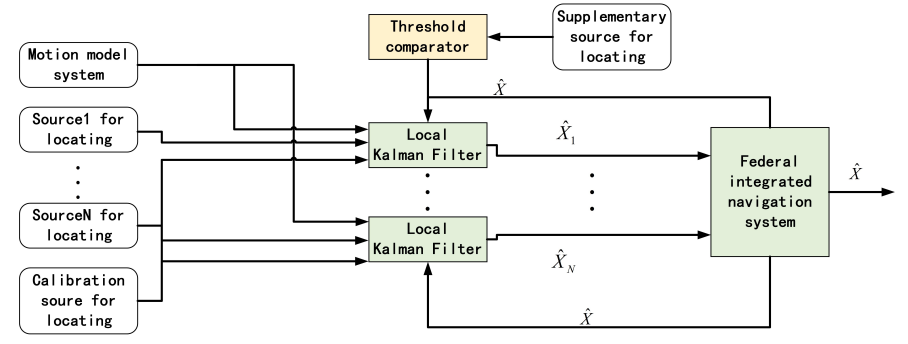

Fig. 10: Federal Kalman filter for sensor fusion in a complex navigation system

performance for non-linear estimation. Particle filtering is widely used in state-of-the-art navigation fusion methods, but due to its computing time restrictions its use should be justified based on the non-linearity of the system.

\section{FACTOR GRAPHS}

The theory of belief functions (BFT), also known as Dempster-Shafer or Evidence theory [67] is an effective theoretical framework for e.g. fusing noisy data. Belief propagation algorithms are normally presented as message update equations on a Factor Graph. Factor Graph is a model combining nonlinear optimization with graph theory. It provides smooth growth and global optimization for estimation. Factor Graph consists of two kinds of nodes, the factor node and the state variable node. If there is an association between two nodes, an edge is built between them. In factor graphs used for fusion, an edge represents both the system model and the measurement model. A famous library based on the Factor Graph theory is G2o [36].

As shown in Fig 11, the $\mathrm{X}, \mathrm{v}, \mathrm{b}$ are the position, velocity and bias state variables and $\mathrm{Z}$ is the vector containing sensor measurements. The optimal state is computed by minimizing the residual error between the estimated state and the state computed using the sensor measurements. An optimization algorithm, such as iSAM2 [29] can be used to deal with minimization problem.

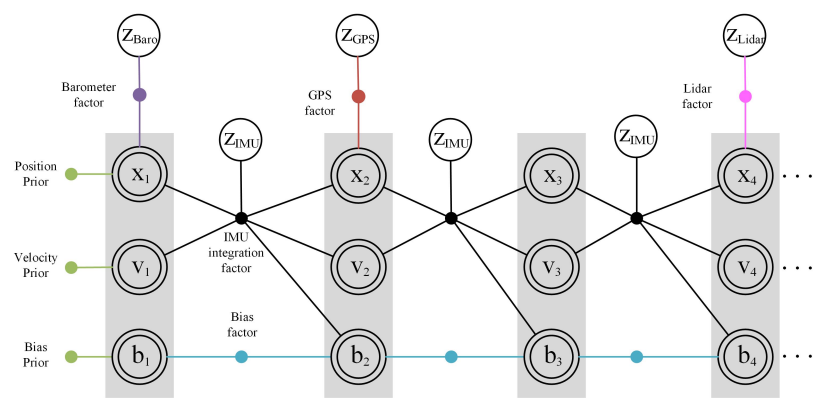

Fig. 11: Factor Graph structure for multi-sensor fusion.

A multi-sensor fusion filter may be implemented as an algorithm finding the minimal residual error using a Factor graph and global optimization. All navigation sensors can be divided into two types of factors as shown in Table III and can be dynamically inserted into an existing factor graph. 
TABLE II: Sensor Classification

\begin{tabular}{|l|l|l|l|}
\hline Sensor & Factor type & Measurement & Respective state \\
\hline INS & binary & $\begin{array}{l}\text { angular velocity } \\
\text { acceleration }\end{array}$ & $\begin{array}{l}\text { attitude, position } \\
\text { speed }\end{array}$ \\
\hline GNSS & unary & $\begin{array}{l}\text { position, speed } \\
\text { sometimes } \\
\text { attitude }\end{array}$ \\
\hline Optical & binary / unary & $\begin{array}{l}\text { distance and angle } \\
\text { rate / location }\end{array}$ & attitude, position \\
\hline LiDAR & binary / unary & $\begin{array}{l}\text { distance and angle } \\
\text { rate / location }\end{array}$ & attitude, position \\
\hline
\end{tabular}

This enables a plug-and-play capability of a sensor fusion framework.

Inertial Measurement Unit (IMU), a sensor providing measurements for INS, operate at much higher rate than other sensor types. Thereupon, fusion can only update the navigation state at the times when non-IMU, such as GNSS data, are available, or when IMU measurements arrive after a certain interval. In the latter case the INS factor is built to connect two sequential navigation states by integrating IMU over the time between the time epochs of the two states.

Compared with IMU measurements, namely angular velocity and acceleration, GNSS is an unary operator involving only one state, position. Cameras and LiDAR could be either binary or unary. If the translation and direction between two frames are used in a graph, the factor is binary. Otherwise, if the location computed by propagating the optical or LiDAR measurements (DR) is used in the framework, the factor is unary. In Fig 11, the framework considers LiDAR and optical (Vision) as unary factors which can provide navigation states directly.

\section{E. ARTIFICIAL NEURAL NETWORKS}

Artificial neural networks are characterized by complex linear or non-linear mapping between input and output with arbitrary precision. Artificial neural networks are complex and composed of a large number of simple components connected to each other. The computation process includes off-line learning and on-line evaluation phases. In the learning process, each neuron conducts regular learning, weight parameter adjustment, and non-linear mapping to achieve training accuracy. Multi-sensor fusion requires mapping of the redundant information with multi-source uncertainty with the fusion result.

\section{MAP MATChING METHODS}

After resolving vehicle's position coordinates they have to be related with a map to make a useful representation. The process is called Map Matching (MM). [62] define MM as a process using road network data to determine the spatial reference of the vehicle's location. It can also be considered as a technique combining electronic map with location information to obtain the real positions of vehicles. Good sources for futher reading are [7], and [62].

The main purpose of a map matching algorithm is to identify the correct road segment a vehicle is travelling and its correct position on that segment [62]. MM algorithms use as an input
TABLE III: Fusion algorithm comparison

\begin{tabular}{|c|c|c|}
\hline $\begin{array}{l}\text { Fusion algo- } \\
\text { rithm }\end{array}$ & Strengths & Weaknesses \\
\hline Kalman filter & $\begin{array}{l}\text { 1. Moderate computation re- } \\
\text { quirements, high efficiency } \\
\text { 2. Optimal estimation for lin- } \\
\text { ear, Gaussian systems }\end{array}$ & $\begin{array}{l}\text { 1. Requires prior probability } \\
\text { and probability independent } \\
\text { hypothesis } \\
\text { 2. Poor performance for non- } \\
\text { linear, non-Gaussian systems }\end{array}$ \\
\hline Particle filter & $\begin{array}{l}\text { 1. Handling of multiple types } \\
\text { of system states } \\
\text { 2. Robustness }\end{array}$ & $\begin{array}{l}\text { 1. Particle degradation } \\
\text { 2. Needs large number of par- } \\
\text { ticles }\end{array}$ \\
\hline Factor graph & $\begin{array}{l}\text { 1. Smooth growth and easy to } \\
\text { expand } \\
\text { 2. Global optimization } \\
\text { 3. Low time synchronization } \\
\text { requirements }\end{array}$ & $\begin{array}{l}\text { 1. Algorithm structure is } \\
\text { complex } \\
\text { 2. High computation require- } \\
\text { ments }\end{array}$ \\
\hline $\begin{array}{l}\text { Artificial } \\
\text { Neural } \\
\text { Network }\end{array}$ & $\begin{array}{l}\text { 1. Strong adaptiveness, fea- } \\
\text { ture classification and map- } \\
\text { ping ability } \\
\text { 2. Possibility for parallel pro- } \\
\text { cessing, efficient }\end{array}$ & $\begin{array}{l}\text { 1. Computationally intensive } \\
\text { 2. Global optimal solution } \\
\text { difficult to find }\end{array}$ \\
\hline $\begin{array}{l}\text { Weighted } \\
\text { fusion } \\
\text { algorithm }\end{array}$ & $\begin{array}{l}\text { 1. Intuitive, easy to under- } \\
\text { stand } \\
\text { 2. Low computation require- } \\
\text { ments, easy implementation }\end{array}$ & $\begin{array}{l}\text { 1. Affected by the quality of } \\
\text { the location source informa- } \\
\text { tion } \\
\text { 2. Weight parameters need to } \\
\text { be estimated via exhaustive } \\
\text { experimenting } \\
\text { 3. Weight parameters are case } \\
\text { dependent }\end{array}$ \\
\hline
\end{tabular}

vehicle's positions and data from high resolution spatial road network maps [63]. These algorithms can be used not only to provide the vehicle's position in the correct segment but also can improve the positioning accuracy if good spatial road network data are available [56].

Here, basic MM methods are briefly discussed, followed by a discussion of their current performance in some relevant scenarios, especially in urban areas.

\section{A. Map Matching methods}

Map matching algorithms range from simple searching techniques to complexes ones using fuzzy logic, Extended Kalman Filter (EKF), and Belief Theory [63]. Approaches can be categorized into four groups:

- Geometrics: those algorithms that make use of geometric information of the spatial road network data considering only the shapes of the links, regardless how they were connected.

- Topological: topological MM algorithms look into the relationship of the entities (points, lines and polygons), as adjacency, connectivity and containment.

- Probabilistic: with the error of the positioning solution, acquired from the sensors (GPS and/or INS and/or DR), elliptical or rectangular confidence regions are built using the error variances of positions. The identification of the roads is made by selecting the segments within this confidence regions.

- Advanced MM algorithms: the advanced algorithms make use of other refined mathematical approaches such as KF or an EKF, Dempster-Shafer's mathematical theory of evidence, a flexible state-space model and a particle filter and interacting multiple model, a fuzzy logic model. 
[63] have reviewed the accuracy obtained using different map matching techniques. The best accuracy obtained at urban and suburban areas among the reviewed methods is around $5.5 \mathrm{~m}$ (95\% cumulative horizontal error) and the correct link identification at over $95 \%$. Map scale, or quality of the map, plays a big role in the MM performances as demonstrated in [83]. MM algorithms matches the positions exactly on the road centreline. Although using the centreline may be desirable situation for many ITS applications, these algorithms introduce large errors in the location estimation, specifically the case of low resolution road network data and therefore is not a good approach for automated vehicles.

\section{B. Map Matching in urban areas}

Digital road maps can be fused with position information for constricting the solution and thereby improving the accuracy [56]. Some algorithms may perform well within suburban areas but not in urban areas and vice versa. Usually, in dense urban areas, map matching algorithms are not capable of reaching positioning requirements for all ITS applications [63] and research on the methods is still required.

[80] developed a MM algorithm based on DempsterShafer's theory of evidence using rule based logical inference system. They matched smoothed GPS positions using a pointo-curve concept. The results suggested a $96 \%$ correct road identification. However, such geometrical method may not behave well in urban areas due to not consideration of the road network topology [63]. [13] proposed a constrained probabilistic interacting multiple model (IMM) to tackle the issue of urban environments. They assumed that the path of the vehicle is known, this way fewer satellites are needed to fix positions. The algorithm uses multiple hypothesis to identify the correct road link. The availability of positions increases in urban areas but the algorithm fails if there is none or only one satellite available. [56] developed a probabilistic MM algorithm and tested the approach integrating a low cost DR sensors and GPS in a EKF. Tests showed that in complex urban roadways and traffic scenarios the algorithm achieved correct matching $100 \%$ of the time. The algorithm also improved the uncertainty of the matched positions from an average of $13 \mathrm{~m}$ (GPS/DR) down to 4 to $5 \mathrm{~m}$ with high-resolution digital map of the road. To overcome noisy navigation positions obtained from GPS in urban canyons, [74] developed a map matching technique based on fuzzy logic. The algorithms showed a high percentage of correct matches and zero percentage of false matches. However, the algorithm took about 30 seconds to fix the first position and also did not consider the map and sensor errors.

MM approaches were developed in the last few years. However, for some ITS applications, these algorithms are not yet capable of supporting the navigation requirements. [63] pointed out the main limitations; initial identification of vehicle positions, matching positioning fixes in complex road lay-out (such as Y-junctions and fly-overs), and performance evaluation in dense urban areas. Although the main purpose of MM algorithms is to accurately display vehicle location on a GIS-based digital map, accurate road map information integrated with other sensors such as cameras, LiDAR, INS, and GNSS can improve positioning accuracy. To fully develop a reliable and robust navigation system, improvements in the algorithms and integration of sensors are still needed to allow safe and continuous navigation in urban and, consequently, in any other type of environment.

\section{CONCLUSION}

This paper reviews the techniques and algorithms needed for developing an accurate and reliable navigation system for autonomous driving. GNSS does not offer the required centimetre level accuracy in most scenarios, especially in urban areas, and definitely not the integrity required from this safety-critical application. Inertial sensors provide measurements that augment the position solution obtained using GNSS, but their weaknesses degraded the fused solution too much to be enough for navigation using GNSS/INS alone. Optical systems provide invaluable information for automated vehicles, also for its navigation. However, optical systems suffer from poor light conditions and other aspects challenging for imaging, especially arctic snow conditions.

We strongly think that the best solution for automated vehicle navigation in Arctic environment is based on multisensor fusion, containing at least a GNSS receiver, INS and optical systems. We think that the optical system are of essential importance for autonomous applications for their accuracy and infrastructure-free nature, however, development of sophisticated computer vision methods for feature and error detection are required. Other critical part in the development is the selection and implementation of the fusion algorithm, in order to obtain a good quality solution. In order to develop a functional traffic ecosystem for the automated vehicles, individual coordinates obtained via positioning have to be related to map information, and therefore the state-of-the-art map matching algorithms were reviewed.

Navigation at the Arctic areas is not addressed enough in the current research. In this paper we underline the open research question to be further addressed in order to obtain a navigation solution with a sufficient performance for autonomous vehicles. To support our conclusions we present some test results obtained using data collected in snowy Arctic environments.

\section{ACKNOWLEDGEMENT}

This paper is co-authored by the IAG Sub-Commission 4.1 WG 4.1.4 'Robust positioning for Urban Traffic' members. Work discussed in Section [V] is partly supported by Shanghai Science and Technology Committee under Grant 17DZ1100803 and NSFC No. 61873163.

\section{REFERENCES}

[1] M. Aeberhard et al., "Experience, Results and Lessons Learned from Automated Driving on Germany's Highways IEEE Intelligent Transportation Systems Magazine, vol.7, no.1, 2015.

[2] Arulampalam, M. Maskell, S., Gordon, N. and T. Clapp. A tutorial on particle filters for online nonlinear/non-Gaussian Bayesian tracking, IEEE trans. on Signal Processing, vol.50, no.2, 2002, pp.174-188.

[3] B. Aumayer, Ultra-tightly Coupled Vision/GNSS for Automotive Applications, $\mathrm{PhD}$ thesis, University of Calgary, 2016. 
[4] E8 - Aurora, the arctic intelligent transport ecosystem: http://www.snowbox.fi/ (Accessed Feb 7, 2018)

[5] Barreau V, Vigneau W, Macabiau C, et al. Kalman Filter based robust GNSS signal tracking algorithm in presence of ionospheric scintillations. ESA Navitec, IEEE, 2014.

[6] K. Bengler, K. Dietmayer, B. Farber, M. Maurer, C. Stiller, and H. Winner, "Three Decades of Driver Assistance Systems: Review and Future Perspectives, IEEE Intelligent Transportation Systems Magazine, vol. 6, no. 4, 2014, pp. 6-22.

[7] J. Berrada and F. Leurent, Modelling Transportation Systems involving Autonomous Vehicles: A State of the Art, Transportation Research Procedia, vol. 27, 2017, pp. 215-221.

[8] Bhuiyan, MHZ (2016) Finland's EGNOS Monitoring and Performance Evaluation (FEGNOS). Final report. Project website: https://fegnos.net/deliverables/. Last accessed 31.8.2018

[9] G. Bresson, Z. Alsayed, L. Yu, and S. Glaser, Simultaneous Localization and Mapping: A Survey of Current Trends in Autonomous Driving, IEEE Transactions on Intelligent Vehicles, vol. 2, no. 3, 2017, pp. 194220.

[10] Bresler, J., Reisdorf, P., Obst, M., Wanielik, G. (2016). GNSS Positioning in Non-line-of-Sight Context - a Survey. 2016 IEEE 19th International Conference on Intelligent Transportation Systems (ITSC), Rio de Janeiro, Brazil, November 1-4, 2016.

[11] K. Stock. Self-Driving Cars Can Handle Neither Rain nor Sleet nor Snow. Bloomberg Businessweek, 17 September 2018.

[12] C. Cappelle, M.E. El Najjar, F. Charpillet, D. Pomorski, Virtual 3D City Model for Navigation in Urban Areas. Journal of Intelligent Robotic Systems, Vol 66, Issue 3, 2012, pp 377-399

[13] Cui, Y., Ge, S. Autonomous vehicle positioning with GPS in urban canyon environments. IEEE Trans. on Robotics and Automation, vol. 19 , no. 1,2003 , pp. $15-25$

[14] A. Della Monica, L. Ruotsalainen, F. Dovis. Multisensor navigation in urban environment. IEEE ION Position, Location and Navigation Symposium (PLANS), 2018.

[15] A. Farman, S.U. Khan, M.Z. Mahmudi, R. Ullah. A Comparison of FAST,SURF, Eigen, Harris, and MSER Features, Intl Journal of Computer Eng. and Information Technology, vol.8, no.6, 2016, pp 100 $-105$

[16] M. A. Fischler and R. C. Bolles, Random sample consensus: A paradigm for model fitting with applications to image analysis and automated cartography, Commun. ACM, vol. 24, no.6, 1981, pp. 381-395.

[17] W. Forstner, A feature based correspondence algorithm for image matching, Int. Arch. Photogrammetry, vol. 26, no. 3, 1986, pp. 150166.

[18] F. Fraundorfer, D. Scaramuzza, and M. Pollefeys, A constricted bundle adjustment parameterization for relative scale estimation in visual odometry, in Proc. IEEE Conf. on Robotics and Automation, 2010, pp. 1899-1904.

[19] J.M. Frahm, P. Georgel, D. Gallup, T. Johnson, R. Raguram, C. Wu, Y.-H. Jen, E. Dunn, B. Clipp, S. Lazebnik, and M. Pollefeys, Building rome on a cloudless day, in Proc. European Conf. Computer Vision, 2010, pp. 368-381.

[20] PV Gakne, M Petovello, Assessing image segmentation algorithms for sky identification, Conf. on GNSS Indoor Positioning and Indoor Navigation, 2015

[21] S. Granshaw, C. Fraser, Eds, Editorial: Computer Vision and Photogrammetry: Interaction or Introspection?, The Photogrammetric Record, Vol 30(149), 3-7

[22] R. Goecke, A. Asthana, N. Pettersson, and L. Petersson, Visual vehicle egomotion estimation using the Fourier-Mellin transform, in Proc.IEEE Intelligent Vehicles Symp., 2007, pp. 450-455.

[23] Gualda D, Urena J U, Garcia-Nunez E. Partially Constrained Extended Kalman Filter for Navigation Including Mapping Information. IEEE Sensors Journal, 2016, PP(99):1-1.

[24] R. Hartley and A. Zisserman, Multiple View Geometry in Computer Vision, 2nd ed. Cambridge U.K.: Cambridge Univ. Press, 2004.

[25] J. Heikkila, Geometric camera calibration using circular control points, in IEEE Transactions on Pattern Analysis and Machine Intelligence, vol. 22, no. 10, pp. 1066-1077, Oct. 2000.

[26] P. Hough, Method and means for recognizing complex patterns, U.S. Patent 3069 654, 1962

[27] Hsu, LT., Jan, SS., Groves, P.D. et al. Multipath mitigation and NLOS detection using vector tracking in urban environments. GPS Solutions, 2015.

[28] Kandepu R, Foss B, Imsland L. Applying the unscented Kalman filter for nonlinear state estimation. Journal of Process Control, 2008, 18(7):753768.
[29] M.Kaess, H. Johannsson, R. Roberts, V. Ila, J. Leonard, and F.Dellaert, iSAM2: Incremental smoothing and mapping using the Bayes tree, Intl. J. of Robotics Research, vol. 31, pp.217-236, 2012.

[30] Kalman R E. A New Approach to Linear Filtering and Prediction Problems. Journal of Basic Engineering Transactions, 1960.

[31] E. Kaplan and D. Hegarty, Eds., Understanding GPS Principles and Applications. Norwood, MA, USA: Artech House, 2006

[32] Q. Ke and T. Kanade, Transforming camera geometry to a virtual downward-looking camera: Robust ego-motion estimation and groundlayer detection, in Proc. Computer Vision and Pattern Recognition (CVPR), June 2003, pp. 390-397.

[33] Koch K. R. Introduction into Bayes-Statistics, Berlin Heidelberg: Springer, 2000

[34] Kong X K X, Nebot E M, Durrantwhyte H. Development of a nonlinear psi-angle model for large misalignment errors and its application in INS alignment and calibration. In Proc. IEEE Conf on Robots and Automation, vol.2, 1999.

[35] P. Koopman and M. Wagner, Autonomous Vehicle Safety: An Interdisciplinary Challenge, IEEE Intelligent Transportation Systems Magazine, vol. 9, no. 1, 2017, pp. 90-96.

[36] Kümmerle R, Grisetti G, Strasdat H, et al. G2o: A general framework for graph optimization, IEEE International Conference on Robotics and Automation. IEEE, 2011.

[37] M. Kutila, P. Pyykönen, A. Lybeck, P. Niemi, and E. Nordin, Towards Autonomous Vehicles with Advanced Sensor Solutions, World Journal of Engineering and Technology, vol. 3, no. 3, 2015, pp. 6-17.

[38] M. Kutila, P. Pyykönen, W. Ritter, O. Sawade, and B. Schaufele, Automotive LIDAR sensor development scenarios for harsh weather conditions, Intelligent Transportation Systems (ITSC), 2016 IEEE 19th International Conf on, 2016, pp. 265-270.

[39] B. Liang and N. Pears, Visual navigation using planar homographies, in Proc. IEEE Int. Conf. Robotics and Automation, 2002, pp. 205-210.

[40] Liu, L. X. Q., Liu, M. L. Z. Map matching algorithm and its application. Intl Journal of Computational Intelligence Systems 2007.

[41] H. Longuet-Higgins, A computer algorithm for reconstructing a scene from two projections, Nature, vol. 293, no. 10, 1981, pp. 133-135

[42] D. Lowe, Distinctive image features from scale-invariant key-points, Int. J. Comput. Vis., vol. 20, no. 2, 2003, pp. 91-110.

[43] Juliette Marais, Sarab Tay, Amaury Flancquart, Cyril Meurie, Weighting with the pre-knowledge of GNSS signal state of reception in urban areas, In Proc. ENC GNSS, 2015.

[44] J Marais, C Meurie, Quantify and improve GNSS quality of service in land transportation by using image processing. In Proc. CNES-ONERA Workshop on Earth-Space Propagation, 2013.

[45] J Marais, Satellite propagation analysis in a masking environment for GNSS applications, IEEE Vehicular Technology Conf, vol. 6, 2005

[46] Mason, S.P. Atmospheric effects on radio frequency (RF) wave propagation in a humid, near-surface environment. Thesis, Naval Postgraduate School, California, 2010.

[47] J. I. Meguro, T. Murata, J. I. Takiguchi, Y. Amano, and T. Hashizume, GPS Multipath Mitigation for Urban Area Using Omnidirectional Infrared Camera, IEEE Trans on Intelligent Transportation Systems, vol. 10 , no. 1,2009 , pp. $22-30$.

[48] MCity test facility: https://mcity.umich.edu/our-work/mcity-testfacility/ (Accessed Feb 9, 2018)

[49] McGehee D.V, Brewer M., Schwarz C., Smith B.W., Review of Automated Vehicle Technology: Policy and Implementation Implications. Report RB28015, Iowa Department of Transportation, 2016.

[50] J. i. Meguro, T. Murata, J. i. Takiguchi, Y. Amano, and T. Hashizume, GPS Multipath Mitigation for Urban Area Using Omnidirectional Infrared Camera, IEEE Trans on Intelligent Transportation Systems, vol. 10 , no. 1,2009 , pp. $22-30$.

[51] Michigan is Waymo's winter wonderland: https://medium.com/waymo/michigan-is-waymos-winter-wonderland9b3cffbb9bab (Accessed Feb 9, 2018)

[52] M. J. Milford and G.Wyeth, Single camera vision-only SLAM on a suburban road network, in Proc. IEEE Int. Conf. Robotics and Automation, 2008, pp. 3684-3689.

[53] H. Moravec, Obstacle avoidance and navigation in the real world by a seeing robot rover, Ph.D. dissertation, Stanford Univ., Stanford, CA, 1980.

[54] Nilsson, J.O., Zachariah, D., Skog, I., and P. Handel. Cooperative localization by dual foot-mounted inertial sensors and inter-agent ranging. EURASIP Journal on Advances in Signal Processing, no. $164,2013$.

[55] D. Nister, O. Naroditsky, and J. Bergen, Visual odometry, in Proc.Int. Conf. Computer Vision and Pattern Recognition, 2004, pp. 652-659. 
[56] Ochieng, W. Y., Quddus, M. A., Noland, R. B. Map-matching in complex urban road networks. Brazilian journal of Cartography, vol. 55, no.2, 2003, pp. 1-14.

[57] Park W-J. and Lee K-L. Rain removal using Kalman filter in video. Intl Conf on Smart Manufacturing Application, 2008.

[58] Pei S-C, Tsai Y-T and Lee, C-Y. Removing rain and snow in a single image using saturation and visibility features. IEEE Intl Conf on Multimedia and Expo Workshops (ICMEW), 2014.

[59] Petovello, M, He, Z. Skyline positioning in urban areas using a low-cost infrared camera. In Proc. of European Navigation Conf, 2016.

[60] Peyret F, Gillieron, P-Y, Ruotsalainen L., Engdahl J. COST Action TU1302. SaPPART White paper: Better use of Global Navigation Satellite Systems for safer and greener transport. Ifsttar, techniques et methodes, TMI 1, 2015.

[61] F. Peyret et al. COST Action TU1302. SaPPART Handbook: Assessment of positioning performance in ITS applications. Ifsttar, techniques et methodes, TMI 2. 2017.

[62] Quddus, M. A., Ochieng, W. Y., Zhao, L., Noland, R. B. A general map matching algorithm for transport telematics applications. GPS solutions, vol.7, no.3, 2003, pp. 157-167.

[63] Quddus, M. A., Ochieng, W. Y., Noland, R. B. Current map-matching algorithms for transport applications: State-of-the art and future research directions. Transportation research part c: Emerging technologies, vol.15, no.5, 2007, pp. 312-328.

[64] Robet, P. P., Evans, B. G., and Ekman, A. Land mobile satellite communication channel model for simultaneous transmission from a land mobile terminal via two separate satellites. International Journal of Satellite Communications, vol.10, no.3, 1992, pp 139-154.

[65] Ruotsalainen, L. Visual gyroscope and odometer for pedestrian indoor navigation with a smartphone. In Proc of Institute of Navigation GNSS+, 2012.

[66] Ruotsalainen L. Vision-Aided Pedestrian Navigation for Challenging GNSS Environments. Doctoral Dissertation, Suomen geodeettisen laitoksen julkaisuja - Publications of the Finnish Geodetic Institute;151. 2013.

[67] Shafer G. A mathematical theory of evidence, vol. 1. Princeton University Press, Princeton, 1976.

[68] Sanroma Sanchez, J., Gerhmann, A., Thevenon, P., Brocard, P., Ben Afia, A., Julien, O. Use of a FishEye Camera for GNSS NLOS Exclusion and Characterization in Urban Environments. ION Intl Technical Meeting, California, 2016.

[69] J. Serafin, E. Olson, and G. Grisetti, Fast and robust 3D feature extraction from sparse point clouds, 2016, pp. 4105-4112.

[70] D. Scaramuzza, F. Fraundorfer, Visual Odometry, Part I: The First 30 Years and Fundamentals, IEEE Robotics and Automation Magazine, 2011

[71] D. Scaramuzza and R. Siegwart, Appearance-guided monocular omnidirectional visual odometry for outdoor ground vehicles, IEEE Trans. Robot. (Special Issue on Visual SLAM), vol. 24, no. 5, 2008, pp. 10151026.

[72] D. Scaramuzza, F. Fraundorfer, and R. Siegwart, Real-time monocular visual odometry for on-road vehicles with 1-point RANSAC, in Proc. IEEE Int. Conf. Robotics and Automation, 2009, pp. 4293-4299.

[73] Suzuki, T., Kubo, N. NLOS GNSS signal detection using fish-eye camera for vehicle navigation in urban environments. In Proc of Institute of Navigation GNSS+, 2014.

[74] Syed, S., Cannon, M. E. Fuzzy logic-based map matching algorithm for vehicle navigation system in urban canyons. In ION National Technical Meeting, vol. 1, 2004, pp. 26-28.

[75] Tabatabaei, A., Mosavi, M.R., Shahhoseini, H.S. et al. Vectorized and federated software receivers combining GLONASS and GPS. GPS Solutions, vol.21, 2017

[76] Sarab Tay, Juliette Marais, Weighting models for GPS Pseudorange observations for land transportation in urban canyons, 6th European Workshop on GNSS Signals and Signal Processing, 2013.

[77] Tao Z. Autonomous Road Vehicles Localization Using Satellites, Lane markings and Vision, Doctoral dissertation, Heudiasyc Laboratory, UMR UTC/CNRS 7253, 2016.

[78] E. R. Teoh and D. G. Kidd, Rage against the machine? Google's selfdriving cars versus human drivers, Journal of Safety Research, vol. 63, 2017, pp. 57-60.

[79] Technical Research Centre of Finland (VTT). Martti, developed for adverse weather conditions, is the first Finnish robot car to challenge snow and ice. ScienceDaily. www.sciencedaily.com/releases/2017/12/171215105156.htm （accessed February 8, 2018).
[80] Dakai Yang, Baigen Cai and Yifang Yuan, An improved map-matching algorithm used in vehicle navigation system, Proc of the 2003 IEEE Intl Confon Intelligent Transportation Systems, vol.2, 2003, pp. 1246-1250.

[81] Yuan G, Yuan K, Zhang H. A Variable Proportion Adaptive Federal Kalman Filter for INS/ESGM/GPS/DVL Integrated Navigation System International Joint Conference on Computational Sciences and Optimization. IEEE, 2011.

[82] X. Zhang, W. Yang, X. Tang, J- Liu. A Fast Learning Method for Accurate and Robust Lane Detection Using Two-Stage Feature Extraction with YOLO v3. Sensors, 2018.

[83] D. Zhao, X. Huang, H. Peng, H. Lam, and D. J. LeBlanc, Accelerated Evaluation of Automated Vehicles in Car-Following Maneuvers, IEEE Transactions on Intelligent Transportation Systems, 2017, pp. 112.

[84] H. Wang, K. Yuan, W. Zou, and Q. Zhou, Visual odometry based on locally planar ground assumption, in Proc. IEEE Int. Conf. Information Acquisition, 2005, pp. 59-64.

[85] G. Welch and G. Bishop, An introduction to the Kalman filter, University of North Carolina at Chapel Hill, NC, Tech. Rep., 1995. 